\title{
Knowing and countering Islamic radicalism: geopolitical and criminological profile and related good practices to combat it
}

\author{
Conoscere e contrastare il radicalismo islamico: profilo geopolitico, criminologico \\ e annesse buone pratiche di contrasto
}

\section{Conocer y contrarrestar el radicalismo islámico: perfil geopolítico y criminológico y buenas prácticas anexas para contrarrestarlo}

\author{
Sara Pacileo \\ Dottoressa in investigazione, criminalità e sicurezza internazionale, Italy
}

\begin{abstract}
In order to assess the complex phenomenon of terrorism, it is necessary to start off by clarifying the terminologies used in the field. For instance, the Occidental terms used to define the most extreme Islamic movements can end up being deviant and imprecise for the ones operating in the field. As a consequence, the Occidental mass media took inspirations from already known terms such as "fundamentalism"; a word much used in the past decades to refer to other religious movements (i.e., catholic fundamentalism, protestant fundamentalism, etc.) and "recycled" to describe the said above Islamic extremism. Nevertheless, there is no agreed in literature upon this topic; the word "radicalism" seems to have the biggest success because it is the one that is most suitable to the way of thinking of the said above movements, the ones that want to go back to their origins. Experts of the Islamic world describe the radicalization as "a process through which a system of extremist values is adopted". Although we have different definitions of the radicalization transition, as it happens in case of a terrorist, we don't have an international definition. In fact, for the experts, it can be really difficult to give a unique definition and assess the motivations that can lead a person to the extremist way of thinking.
\end{abstract}

\section{RIASSUNTO}

Il punto di partenza necessario per un'accurata analisi sul mondo del terrorismo si ritrova nella puntualizzazione delle terminologie utilizzate e, soffermandoci su quelle occidentali utilizzate per definire i movimenti islamici più estremi e militanti, alcune di esse sono risultate imprecise e devianti per coloro che valutano e operano nella realtà. Ciò risulta essere una conseguenza di una valutazione attuata ad un mondo poco conosciuto e che man mano stava, in maniera repentina, acquisendo sempre più clamore. Di conseguenza, i mezzi di comunicazione occidentali hanno attinto a termini già conosciuti: "fondamentalismo", "integralismo" sono termini che sono stati già utilizzati in passato (integralismo cattolico o fondamentalismo protestante) e che sono stati "riciclati" per descrivere i suddetti movimenti islamici più estremi. Tuttavia, i termini sopracitati non riflettono a pieno la realtà. Gli esperti non ne condividono l'utilizzo. Maggiore successo sembra avere il termine "radicalismo" che risulta essere più consono e attinente al pensiero dei suddetti movimenti, ovvero quello di volere un "ritorno alle radici". ${ }^{1}$ Diversi conoscitori del mondo islamico, definiscono la radicalizzazione come "il processo attraverso il quale si adotta un sistema di valori estremista, inclusa la volontà di usare, supportare o facilitare la violenza come metodo per il cambiamento sociale". ${ }^{2}$ Nonostante diverse definizioni del concetto di radicalizzazione, come nel caso del terrorismo, non esiste una definizione internazionale. Difatti, per gli studiosi della materia risulta essere difficile tanto darne una definizione univoca quanto stabilire le motivazioni che avvicinano l'individuo alla stessa idea estremista.

\section{RESUMEN}

El punto de partida necesario para un análisis certero del mundo del terrorismo se encuentra en la aclaración de la terminología utilizada y, deteniéndonos en la terminología occidental utilizada para definir los movimientos islámicos más extremos y militantes, parte de ella ha resultado inexacta y desviada para quienes evalúan y operan en la realidad. Esto resulta ser la consecuencia de una evaluación realizada sobre un mundo poco conocido y que, poco a poco, fue adquiriendo más y más clamor. En consecuencia, los medios de comunicación occidentales han recurrido a términos ya conocidos: "fundamentalismo", "integralismo" son términos que ya se han utilizado en el pasado (integralismo católico o fundamentalismo protestante) y que se han "reciclado" para describir los movimientos islámicos más extremos antes mencionados. Sin embargo, los términos anteriores no reflejan totalmente la realidad. Los expertos no están de acuerdo con su uso. El término "radicalismo" parece más acertado y parece más adecuado y pertinente al pensamiento de los movimientos mencionados, es decir, querer una "vuelta a las raíces". Varios conocedores del mundo islámico definen la radicalización como "el proceso mediante el cual se adopta un sistema de valores extremista, incluida la voluntad de utilizar, apoyar o facilitar la violencia como método de cambio social". A pesar de las diferentes definiciones del concepto de radicalización, como en el caso del terrorismo, no existe una definición internacional. De hecho, para los estudiosos del tema, es tan difícil dar una definición inequívoca como establecer las motivaciones que acercan al individuo a la misma idea extremista.

Rover V, rischio sociale e sicurezza nazionale: il radicalismo islamico. Analisi della sua origine, teorizzazioni e possibili riflessi sulla sicurezza nazionale. Gnosis n.2. http://gnosis.aisi.gov.it/sito/Rivista2.nsf/Serv Navig/4
2 Allan, H.; Glazzard A., Jesperson, S., Reddy-Tumu, S., Winterbotham E. Drivers of violent extremism, hypotheses and literature review, Royal United Service Institute (RUSI), 2015. https://bit.ly/ 2LTgeHv 


\section{Definizione di terrorismo}

'E' sempre più difficile definire giuridicamente la minaccia terroristica, come dimostra il lavoro in corso alle Nazioni Unite"3. Letteralmente il termine terrorismo va a significare "indurre terrore", definizione che non delinea il fenomeno e rende i suoi confini molto incerti: il terrore può essere indotto anche da fenomeni estranei al terrorismo. Come anticipato in precedenza, anche le Nazioni Unite stanno cercando una definizione univoca del termine ma, non sono arrivati a definire il fenomeno poiché alcuni paesi si sono opposti a considerare "terroristi" i combattenti per la libertà. Il primo punto che quindi va sottolineato è che il terrorismo non può essere definito all'interno di un sistema di valori, cioè non si possono dare "giudizi di valore". L'ONU ha definito un "atto di terrorismo" un atto di violenza contro cittadini innocenti. Anche questa definizione risulta incompleta poiché non va a definire la categoria di "cittadini innocenti": all'interno non vengono inseriti militari, poliziotti, magistrati etc., spesso vittime di atti di terrorismo.

$\mathrm{Nel}$ 1994, in occasione della risoluzione 49/60 dell'assemblea generale dell' $O N U$, all'interno dell'articolo 3 si va a stabilire una definizione di terrorismo:

"Gli atti criminosi volti a provocare uno stato di terrore tra la popolazione, un gruppo di persone o determinate persone per fini politici sono, in ogni circostanza, ingiustificabili, quali che siano le considerazioni politiche, filosofiche, ideologiche, razziali, etniche, religiose o di altro tipo che possano essere invocate per giustificarli".

Brevemente, il terrorismo può essere classificato in quello che potremmo definire "domestico" ed "esterno".

Per quanto riguarda il primo, ci soffermeremo brevemente; Parliamo di un tipo di terrorismo creato da gruppi di matrice nazionale o comunque ben radicati all'interno di uno Stato in cui operano. A sua volta si può dividere in "strategico", "diffuso" o "indotto". Il primo fa riferimento a gruppi ben strutturati (come, ad esempio, le BR); Il secondo si colloca alla periferia dei gruppi strategici ed infine il terzo descrive piccoli gruppi con scarsa organizzazione.

In riferimento alla seconda categoria, di nostro interesse, abbiamo un'ulteriore suddivisione in:

a) Terrorismo internazionale: è una forma di terrorismo nella quale si colpiscono obiettivi al di fuori del proprio paese. Lo scopo risulta essere sia quello di indebolire il sostegno che questi paesi danno ai loro avversari personali sia quello di terrorizzarli; vengono, così, inquadrati nell'area del nemico. Questa definizione può essere applicata ad al- Qaeda e in parte alll'ISIS.

b) Terrorismo transnazionale: in questo caso parliamo di una forma di terrorismo in cui l'attentato vede coinvolgere un paese "ospite"; si cerca un bersaglio all'interno del Paese, lo si attacca, ma in realtà si vuole mirare ad indebolire non il Paese stesso, ma un Paese terzo. Uno dei casi più esplicativi risulta essere quello dei curdi negli anni '70 in cui hanno colpito uffici del turismo turchi in tutta Europa. (L'obiettivo, quindi, risultava essere la Turchia e non il paese ospite che poteva variare nel contesto europeo).

c) Di stato: è una forma di terrorismo che vede operare terroristi o agenti di una potenza straniera ai danni di un altro paese. In questo caso si agisce "dall'interno". In questa forma di terrorismo, nel momento in cui si accerta che la cellula operativa è formata dal personale inviato, la risposta può avvenire con una chiusura diplomatica per arrivare, in caso di prove chiare, anche ad una risposta militare. d) Sponsorizzato da stati: In questo caso si parla di Stati che sponsorizzano il terrorismo fornendo armi o denaro o, eventualmente, istigando il gruppo terrorista.

Di estrema attinenza risulta essere il pensiero di esperti, i quali, hanno osservato come anche il fenomeno del terrorismo sia mutato nel tempo:

"La questione viene ripetutamente sollevata in merito alla definizione del "terrorismo", con particolare riferimento ai concetti di "guerra" e "lotta di liberazione". Cominciamo col prendere atto del fatto che la minaccia del nuovo terrorismo è qualitativamente diversa dalle tradizionali minacce con cui ci si è confrontati nell'ultimo mezzo secolo. Le coordinate per definirla e affrontarla non sono più quelle degli stati nazionali, ma quelle delle visioni del mondo e degli interessi economici. Le guerre oggi tendono ad avere carattere reticolare e diffuso, con protagonisti tra loro asimmetrici: stati, terroristi, bande armate, gruppi etnici eccetera."4

\section{Nemico "fluido" e superamento del concetto "Stato-Nazione"}

Per poter comprendere a pieno il fenomeno e porre le basi per poterlo contrastare è importante analizzare il "nuovo nemico" nella sua percezione, e il concetto, o meglio, il superamento del concetto di "Stato-Nazione". Non a caso il termine "fluido" risulta attribuito alla nuova tipologia di nemico. Il primo a parlare di "liquidità" nella postmodernità è stato il sociologo Zygmunt Bauman, tant'è che è proprio dalla sua visione che si svilupperà il concetto di "nemico fluido": "Il liquido, lasciato a sé stesso, tende a scorrere in mille rivoli, cambiare direzione ad ogni ostacolo, sino ad annullarsi e perdere ogni distinzione. L'esito è la dissoluzione in milioni di gocce, atomi liquidi privi di valore, direzione, centro". 5

Il concetto di base risulta essere in realtà molto semplice, ma per niente banale, se vogliamo esaminare l'argomento in ogni suo aspetto. Definendo il terrorismo come un "nemico fluido", si vuole far riferimento ad un nemico che varia dalla vecchia concezione, non essendo delineato da confini territoriali: se nella storia, le guerre sono state caratterizzate da nemici ben definibili e con un proprio territorio circoscritto, ad oggi il terrorismo abbandona tutte queste caratteristiche.

Al giorno d'oggi il nostro nemico può colpire chiunque, in qualunque momento e in qualsiasi spazio.

Questa riflessione, a sua volta, porta necessariamente a riflettere anche sul concetto di stato- nazione e sul paradigma vestfaliano. Se non esiste più un nemico definito, può ancora esistere la dicotomia interno- esterno?

Il nemico fluido non è più colui che si trova lontano dal nostro Paese, colui che agisce "fuori" dal territorio, anzi, ad oggi, il nostro nemico può trovarsi e agire più vicino di quanto si possa immaginare.

Come superare il paradigma vestfaliano, il concetto di "StatoNazione"?

3 Sbailò C., terrorismo contemporaneo. Nuove minacce e vecchi diritti. Gnosis 3/2006, 2016. http:/gnosis.aisi.gov.it/gnosis/Rivista8.nsf/ Serv Navig/11

4 Sbailò, Gnosis, rivista italiana di Intelligence, 3/2016. http://gnosis.aisi.gov.it/gnosis/Rivista8.nsf/ServNavig/11

5 Pecchioli, mondo liquido: l'uomo in bottiglia. http://www.unavox.it/ ArtDiversi/DIV3347_Pecchioli_Mondo_liquido.html 4.10.2020 
In alternativa si potrebbe parlare di "non- stato" ovvero di deterritorializzazione; anche in questo caso ci troveremmo dinnanzi ad una dicotomia, come quella interna/esterna, di territorio/non territorio. Il tutto ci riporta al paradigma. Potremmo, piuttosto parlare di "iper-territorializzazione": concetto dettato dal fatto che, come accennato in precedenza, i confini iniziano a non essere più quelli fisici. (sia dal punto di vista del nemico fluido, sia dal punto di vista della difesa).

La comprensione di questa prospettiva risulta necessaria e pone le basi per la questione della "difesa", poiché solo alla venuta comprensione della tipologia di nemico che abbiamo di fronte, ci si potrà difendere da esso.

\section{Il jihadismo ai tempi del COVID-19}

L'attuale emergenza sanitaria dovuta all'espandersi a livello mondiale del così chiamato "coronavirus" ha portato ogni individuo a dover stravolgere la propria vita e riorganizzarla. Le abitudini di un bambino non sono più le stesse, come non lo sono quelle di un commerciante o di un Presidente; tutti si sono dovuti adattare.

Alla luce di ciò, anche le organizzazioni terroristiche hanno dovuto riorganizzarsi e reagire all'emergenza sanitaria; in questo paragrafo, si cercherà di indagare sul loro spirito di adattamento in risposta al virus e soprattutto di analizzare la loro interpretazione in relazione al fenomeno.

Sicuramente, anche essi, hanno dovuto limitare la loro presenza sul territorio ed hanno utilizzato le medesime linee guida diffuse dall'Organizzazione Mondiale della Sanità.

Questo nuovo scenario ha portato, ancora una volta, a marcare le differenze tra al-Qaeda e l'ISIS; diversi, infatti, sono stati gli approcci utilizzati dalle due organizzazioni; la prima, con un approccio molto più vago e generale, ha pubblicato un documento sul proprio canale internet, " $A s-S a h a b$ ", nel quale afferma che il COVID-19 è la giusta punizione divina per gli infedeli, soprattutto per l'Occidente.

L'ISIS, invece, all'inizio dell'emergenza ha pubblicato sulla propria rivista $(\mathrm{Al}-\mathrm{Naba})$ delle linee guida molto precise per i propri affiliati, aggiungendo anche una componente religiosa poiché, le suddette sono direttamente ispirate dal Profeta ${ }^{6}$.

Una particolare attenzione, però, può essere rivolta alle linee guida in riferimento agli spostamenti: ai fedeli, era stato detto di non attuare alcun tipo di spostamento, anche se questi ultimi si trovavano in zone rosse. Alcuni, hanno interpretato queste limitazioni in riferimento alle attività terroristiche e non alla libertà di movimento dell'individuo; come un messaggio per porre una sospensione alle operazioni e di conseguenza una sospensione a quegli spostamenti al fine di compiere un attentato terroristico ${ }^{7}$.

Per i jihadisti il coronavirus può essere visto sia come una punizione che una benedizione. Il virus in circolazione non attua distinzione, di conseguenza anche i jihadisti sono a rischio come qualsiasi altra persona e sono limitati nelle loro attività; d'altro canto però, diverse sono le dinamiche che hanno portato a dei vantaggi alle organizzazioni terroristiche: basti pensare al caos generale, alle difficoltà a livello sanitario ed economico che gli Stati Occidentali stanno fronteggiando per capire che il livello di attenzione dei governi e media occidentali si è abbassato notevolmente in relazione alla lotta al fenomeno.

Allo stesso tempo, se si analizzano le statistiche degli attacchi che l'occidente ha ricevuto nel periodo che va dall'inizio dell'epidemia a ottobre, si può osservare come essi siano decisamente diminuiti, sia per l'aumento dei controlli per garantire il rispetto delle norme
anti-COVID, sia perché sono venuti meno possibili obiettivi da colpire, in assenza di assembramenti. Questo ha portato, ancor di più, a distogliere l'attenzione dal problema del terrorismo.

Un altro aspetto a cui non si sta dando il giusto peso, in questo momento così particolare, è l'aumento esponenziale di internet durante il lockdown; anche coloro che prima non amavano utilizzare internet per compere, per leggere le notizie quotidiane o semplicemente non sapeva utilizzare le nuove tecnologie, con l'emergenza in corso, sono stati costretti a dover affacciarsi al mondo dell'internet. Ad approfittare di ciò, sono state le organizzazioni jihadiste, esportando la loro idea sull'epidemia, per dare direttive e soprattutto, attuare propaganda e diffondere il loro messaggio a nuovi adepti. Tutto ciò potrà portare a delle conseguenze molto serie, considerando che ad essere colpite da questa nuova situazione, sono soprattutto le fasce della popolazione più deboli che, in relazione al fenomeno che si sta analizzando, sono quelle persone più vulnerabili e inclini ad una radicalizzazione ${ }^{8}$.

Un ultimo aspetto, non meno preoccupante, risulta essere un possibile utilizzo del virus, da parte dei terroristi, come arma biologica. Può sicuramente risultare controproducente per gli stessi e ad oggi, non ci sono dei precisi episodi che possano ricondurre alla diffusione del virus da parte di radicali, ma l'idea non è poi così lontana dalla realtà.

Ci sono stati degli episodi in vari Paesi occidentali, di persone che hanno starnutito o tossito su agenti di polizia e così via, ma non sono riconducibili al fenomeno.

Allo stesso tempo, però, non sono mancate, all'interno della propaganda via internet, frasi di incitamento alla diffusione del virus nelle zone degli infedeli e tra le forze di sicurezza, difatti, è stato arrestato un islamista, il 16 aprile 2020, dalle forze di polizia tunisine poiché stava progettando di diffondere il virus tra le forze di difesa. In ogni modo, l'utilizzo del virus come arma, al momento, risulta essere limitato alla sfera di internet ${ }^{9}$.

Il rischio che in questo momento corre l'Occidente è quello di porre il focus interamente sull'epidemia e sottovalutare il nemico del terrorismo internazionale, che rimane dietro l'angolo.

\section{Attentati di Nizza e Vienna 2020}

Alla luce dei recenti risvolti, sembrerebbe che lo scenario sopracitato si stia manifestando: i giorni 30 ottobre e 2 novembre 2020 hanno avuto luogo due attentati terroristici, rispettivamente a Nizza e Vienna.

Brevemente il resoconto dei due attentati:

A Nizza, l'attentato è avvenuto nella cattedrale di Notre Dame; l'attentatore ha ucciso tre persone. Si tratta di un tunisino di 21 anni, arrivato in Europa pochi mesi fa, precisamente a Lampedusa. In questo episodio non ci sono state rivendicazioni e ci sono ancora delle indagini in corso. Il sindaco della città riporta alcune infor-

6 Palladini G., il terrorismo ai tempi del covid-19, Eurobull, 2020. https://www.eurobull.it/il-terrorismo-ai-tempi-del-covid-19?lang=fr

7 Dacrema E., Il jihadismo ai tempi del coronavirus. Ispionline, 2020 https://www.ispionline.it/it/pubblicazione/il-jihadismo-ai-tempi-delcoronavirus- 25635

8 Cossiga A., Per i jihadisti il coronavirus è una punizione e una benedizione. Limes, 2020 https://www.limesonline.com/coronavirus-radicalizzazione-islam-jihadismo-stato-islamico/118036

9 Giacalone G., I terroristi possono sfruttare il coronavirus? Insideover, 2020. https://it.insideover.com/terrorismo/i-terroristi-possonosfruttare-il-coronavirus.html 
mazioni relative all'attentatore, una delle quali rivela che, l'uomo mentre veniva medicato dopo essere stato ferito dalla polizia, ripeteva in continuazione "Allah Akbar". ${ }^{10}$ Inoltre, nello stesso giorno, sembrerebbe che altri due uomini, in zone diverse del paese, avrebbero provato ad attuare un attentato, con le stesse modalità. Ed è per questo motivo che la Francia ha attuato di nuovo i massimi livelli di sicurezza antiterrorismo. ${ }^{11}$

A Vienna, l'attentato sembra portare alla luce, le conseguenze che sopra erano state ipotizzate in relazione al coronavirus e terrorismo; Vienna, è sempre stata considerata una delle città più sicure d'Europa e improvvisamente è divenuta protagonista di un attentato, svolto nel pieno centro del paese. Si contano cinque vittime e sedici feriti, uccise da un uomo che ha iniziato a sparare per le vie di Vienna. Ancora da stabilire la presenza di complici. L'ISIS il giorno dopo ha rivendicato l'attacco attraverso i suoi canali di propaganda, definendo l'attentatore "un soldato del Califfato". Kujtim Fejzulai è il nome dell'uomo, ucciso dagli agenti di polizia; circa un anno prima è stato condannato a 22 mesi di carcere per aver cercato di andare in Siria per unirsi allo Stato Islamico. ${ }^{12}$

Probabilmente, la scelta di colpire Vienna il 2 novembre 2020, non è stata casuale: l'organizzazione terroristica ha sfruttato l'inevitabile assembramento data l'ultima sera di via libera, poiché il giorno dopo sarebbe scattato il lockdown.

Ci troviamo di fronte ad un avanzamento del terrorismo che sfrutta la pandemia per essere meno controllato ed agire? La concentrazione verso il contenimento della pandemia ha portato Vienna a non essere più una delle città più sicure d'Europa? I disagi dati dalla pandemia avranno aumentato il divario e il risentimento sociale portando all'incremento di questi atti violenti?

\section{Quadro psicologico e criminologico del terrorista}

Comprendere il perché dell'adesione di determinati soggetti alle idee estremiste è un processo molto complesso. Diversi sono $\mathrm{i}$ fattori che vanno ad influenzare e incidere sulla suddetta scelta: fattori culturali, religiosi, sociali, personali e politici.

Un approccio da un solo punto di vista rischierebbe di non dare una completa visione del fenomeno, che risulta complesso ed articolato. In virtù di ciò, è importante dedicarsi alla sfera psicologica e criminologica della figura in sé del terrorista, attuando un approccio multidisciplinare che punti a comprendere le ragioni che portano un individuo a aderire al radicalismo e sacrificare la sua vita per la causa.

In altri termini, si va ad attuare sia "un'analisi di tipo strutturale", con l'obiettivo di inquadrare tutti i possibili fattori di radicalizzazione, dal contesto sociale (come l'assenza di lavoro e disuguaglianze) a quello identitario (razzismo, xenofobia, segregazione sociale) o di disgregazione sociale, sia "un'analisi individualizzata", in modo da studiare anche il percorso del singolo. ${ }^{13}$

\section{Suicidio, martirio e autolesionismo}

Una domanda che spesso ci si pone è come mai nel caso dei così detti "terroristi kamikaze", ${ }^{14}$ la sfera religiosa non entri in contrasto con l'azione dell'individuo.

Questo perché nell'Islam il suicidio è rigorosamente proibito. La motivazione si pone alla base del pensiero secondo cui, l'unico a poter scegliere della vita del credente è Dio; con lo scegliere di agire autonomamente sulla propria vita, ponendone la parola fine, si mette in discussione il diritto che spetta solo a Dio. L'azione del suicidio, inoltre, va a manifestare la mancanza di "sabr", intesa come la capacità di saper affrontare e resistere alle difficoltà della vita. L'islam non accetta in alcun modo la violenza attuata su sé stessi, per questo motivo non accetta nemmeno l'autolesionismo.

In un caso, però, il suicidio non viene considerato tale: quando si parla di "martirio"; in questa circostanza, al contrario, si parla di prestigio sia a livello religioso che sociale. Nel mondo sunnita non esiste una netta distinzione tra chi può essere considerato legittimamente un martire e chi invece un terrorista suicida. ${ }^{15}$

Eppure, continua a risultare un paradosso. Le parole di Maometto sono ben chiare in relazione al suicidio:

“chiunque si uccida con un'asta di ferro, è destinato a continuare a tenere l'asta in mano e a dilaniarsi per quel mezzo l'addome con il fuoco dell'inferno, dove rimarrà in eterno. Chiunque beva del veleno e si uccida, lo berrà nel fuoco dell'inferno, dove rimarrà in eterno. Chiunque si getti da una montagna e si uccida, sarà gettato nel fuoco dell'inferno, dove rimarrà in eterno". ${ }^{16}$

In realtà, il profeta definisce la linea di confine tra "martire" e "terrorista suicida". Il martire è colui che muore in battaglia, senza premeditazione, risultante di un imprevisto. Il terrorista suicida è colui che consapevolmente, per la causa, decide di privarsi della propria vita.

Allora, perché i terroristi, nonostante sostengano di essere portatori della parola dell'Islam, decidano di attuare gli attentati con attacchi suicida, avendo anche la possibilità di compierli in altri modi?

Alcuni autorevoli esperti ${ }^{17}$ sostengono che per il terrorista la morte non risulta essere un pegno, qualcosa da pagare per una causa più grande o un mezzo per rendere l'attacco più efficace, ma semplicemente il jihadista brama la sua morte e vuole arrivare ad essa tanto quanto quella delle sue vittime. A rendere tutto più chiaro è il pensiero attribuito a Bin Laden: "noi amiamo la morte come voi amate la vita". 18

Alla domanda, sembra esserci un'unica soluzione intellettuale per dare una risposta al paradosso sopracitato:

"Per conciliare il suicidio e la fede religiosa dei jihadisti - i quali, non c'è dubbio, sono convinti di andare in paradiso compiendo l'atto - serve citare l'attesa dell'apocalisse. Per loro siamo alla fine dei tempi, e alla fine dei tempi saltano tutte le regole. È un aspetto che unisce lo Stato islamico nella sua dimensione locale in Medio Oriente e il jihad globale dei terroristi. Il primo insegue il progetto teologicamente impossibile di ristabilire il Califfato di Maometto, laddove il profeta non può tornare e sarebbe eretico dal punto di vista dello stesso Islam pensare che ne arrivi un altro dopo di lui. I secondi fanno appello a Daesh sacrificando nelle proprie azioni suicide qualsiasi logica strategico-militare, il calcolo cinico

10 "Dio è il più grande", espressione utilizzata dai jihadisti prima di compiere un attentato.

11 https://www.corriere.it/esteri/20 ottobre 29/francia-settantennedecapitato-cattedrale-nizza-14d8028e-19c2-11eb-bdd5-3ce4cb03ccdf shtml 30.10.20

12 https://www.ilpost.it/2020/11/03/attentato-vienna-morti-feriti/

13 Guolo R., il dibattito sulla radicalizzazione nelle scienze sociali. Guerini e Associati, Milano, 2017. p.75.

14 Attentatore che perde volontariamente la vita nell'azione terroristica che compie.

15 Denaro R., Sillabo: conoscere l'islam per contrastare il radicalismo, 2018, p.68.

16 Giangrande A., Gesù Cristo vs Maometto e l'islamizzazione del mondo, 2016. p. 297

17 Roy O., Esperto di islamismo e fondamentalismo religioso, cerca di dare una risposta

18 Roy O, Generazione isis: chi sono i giovani che scelgono il Califfato e perché combattono l'Occidente. Milano, 2017 
e non metafisico che guidava invece i kamikaze giapponesi nella Seconda guerra mondiale o quelli del Pkk curdo e altre organizzazioni, e ogni coerenza religiosa. La resa dei conti finale di Dabiq, la località siriana che dà il nome alla rivista di Daesh e in cui è previsto lo scontro dell'Apocalisse, svuota di significato ogni progetto o regola. È inutile razionalizzare, cercare di spiegare perché Daesh scelga certi obiettivi piuttosto che altri. Daesh è contro tutto e a favore di niente, non crede neppure nella sua distopia". ${ }^{19}$

\section{Approccio psicologico, sociologico e criminologico al fenomeno}

Da un punto di vista della psicologia il compiere un attentato terroristico è la risultante di percorsi biografici. Per questo motivo gli studiosi trovano difficile definire un preciso identikit psicologico del terrorista, poiché le motivazioni che spingono l'individuo ad unirsi al radicalismo possono essere differenti.

Sicuramente la psichiatria ha escluso totalmente la presenza di malattie mentali nei soggetti presi in esame; tutti gli studi hanno dimostrato che non ci sono argomentazioni valide per definire il terrorista un "folle". Da un punto di vista psichiatrico la mente del terrorista è "normale". ${ }^{20}$

Si è cercato, di seguito, di analizzare la figura del terrorista attingendo dalle teorie psicologiche, criminologiche e sociologiche.

Anche se da un punto di vista psichiatrico e psicologico i dati effettivi risultano essere scarsi, da una prima panoramica data da diverse ipotesi, sembra che gli esperti concordino nel definire il fenomeno dell'avvicinamento al terrorismo la risultante di uno sviluppo delle diverse fasi della crescita, deviato da influssi non perfettamente ortodossi. Di seguito alcune teorie:

- Alcuni esperti sottolineano come spesso, la scelta di unirsi al radicalismo sia condizionata da predisposizioni individuali causate da vissuti infantili o adolescenziali che hanno portato ad un trauma, andando ad incidere sulla soglia di tolleranza allo stress interpersonale del soggetto. ${ }^{21}$

- Altri, affermano che l'atteggiamento del terrorista può essere definito "totalizzante" poiché il soggetto considera la dottrina politica e la visione ideologica dell'esistenza punti imprescindibili per la sua stessa esistenza e, di conseguenza, senza di esse la sua vita potrebbe anche cessare. Il comportamento del terrorista è la risultate di due fattori: il fanatismo e una visione paranoide del mondo; mentre il primo fattore va ad agire sulla lettura che il soggetto dà al presente, il secondo è il fattore che crea coerenza tra il pensiero e l'agire del terrorista. La paranoia crea un processo di radicalizzazione nel soggetto che lo porta a legittimare le sue reazioni nei confronti della realtà, vista estranea e minacciosa. ${ }^{22}$

- Il Center for Strategic and International Studies (CSIS) ha compiuto uno studio sul processo di radicalizzazione, arrivando a distinguere diversi elementi che possono portare l'individuo ad avvicinarsi al mondo del terrorismo: la presentazione delle idee radicali, poste come filtro per comprendere la realtà; i fattori sociologici, che spingono l'individuo a condividere le suddette idee; i fattori psicologici che portano il soggetto a ritenere che la violenza sia l'unico modo per poter promuovere $\mathrm{i}$ suoi ideali. ${ }^{23}$

- Per comprendere meglio il processo di radicalizzazione, alcuni autori, ${ }^{24}$ hanno introdotto il concetto di "fattori di rischio"; nel momento in cui nell'individuo sono presenti i suddetti elementi, con la propaganda e la persuasione è più probabile che il soggetto sia propenso alla radicalizzazione. Gli autori evidenziano come alcuni tratti di personalità siano comuni nel terro- rista (narcisismo e paranoia) e anche come, spesso, abbiano condiviso le stesse esperienze: crescita tra le seconde o terze generazioni di immigrati; soggiorno in carcere; problemi in famiglia; difficoltà ad inserirsi all'interno della società o viaggi in zone con alta presenza di gruppi terroristici.

Uno strumento interessante della psicologia, utilizzato dagli esperti della materia, risulta essere il "test della torre di Londra": è un test neuropsicologico avente come scopo la valutazione delle funzioni esecutive, cioè la capacità di un soggetto di poter compiere una determinata azione. Questo perché alla base della psicologia risulta esserci la possibilità di misurare il comportamento umano e, di conseguenza, misurare se un soggetto è capace di premeditare un'azione.

Suddetto test, ad esempio, è stato utilizzato nel processo riguardante Eric Matthew Frein, ${ }^{25}$ per comprendere se il soggetto fosse stato capace di poter progettare l'attentato da egli stesso compiuto. Frein ha passato il test, dimostrando di essere in grado di calcolare le azioni anticipatamente.

"La scelta terroristica, dunque, verrebbe a configurarsi non come una condizione ma come un processo. Dato che ogni percorso è diverso e influenzato da molteplici fattori, la radicalizzazione non deve essere considerata come «il prodotto di una singola decisione, ma il risultato finale di un processo dialettico che spinge progressivamente un individuo verso un impegno alla violenza nel tempo". ${ }^{26}$

Oltre ad un approccio psicologico, anche la sociologia ha dato un contributo per arrivare a comprendere la motivazione per cui un individuo decide di avvicinarsi al mondo del terrorismo. Principalmente, Borum, distingue tre correnti ideologiche: la Social Movement Theory, la Social Psychology e la Conversion Theory.

Per quanto riguarda la Social Movement Theory, è una scuola di pensiero nata negli anni Cinquanta, la quale sostiene che i movimenti radicali siano la risultante di comportamenti irrazionali inseriti in precarie condizioni ambientali. Queste condizioni porterebbero l'individuo a vivere passivamente e di conseguenza, a aderire passivamente ai movimenti. Con l'avanzare degli studi, gli esperti hanno convenuto come anche i legami sociali e le relazioni giochino un ruolo fondamentale nel processo di radicalizzazione ${ }^{27}$.

La Social Psychology, infatti, concentra i propri studi soprat-

19 Intervista ad Oliver Roy. 2.11.20. https://espresso.repubblica.it/ plus/articoli/2017/06/13/news/1-islam-condanna-il-suicidio-e-questo-ilparadosso-del-terrorimo-jiadhista-1.304208

20 Munizio C., nella mente del terrorista, l'ambizione di un passaggio alla storia. Le ragioni della follia; intervista al Presidente del Gruppo Italiano di Psichiatria (SIP) (https://www.insalutenews.it/in-salute/nellamente-del-terrorista-lambizione-di-un-passaggio-alla-storia-le-ragionidella-follia/)

21 Mastronardi V, Leo S, Terroristi Newton \& Compton 2005.

22 Vercelli C., che cos'è la radicalizzazione. Note di riflessione a margine di un processo di politicizzazione di alcune minoranze islamiche. p.10 http://webcache.googleusercontent.com/search?q=cache:i1tJ-8DUTMcJ: siba-ese.unisalento.it/index.php/eunomia/article/download/16166/13946+ $\& \mathrm{~cd}=1 \& \mathrm{hl}=\mathrm{it} \& \mathrm{ct}=\mathrm{clnk} \& \mathrm{gl}=\mathrm{it}$

23 Laurano e Anzera, L'analisi sociologica del nuovo terrorismo tra dinamiche di radicalizzazione e programmi di de- radicalizzazione. 2017 p.3 .https://journals.openedition.org/qds/1792

24 Tra cui citiamo, Leistedt, professore di psicologia criminale.

25 Ricercato per l'omicidio di un agente della Pennsylvania ed inserito nella lista dei 10 criminali più ricercati d'America.

26 Borum, radicalization into Violent Extremism I: A Review of Social Science Theories, 2011. p.15 (https://scholarcommons.usf.edu/ jss/vol4/iss $4 / 2 /$ )

27 Laurano e Anzera, L'analisi sociologica del nuovo terrorismo tra dinamiche di radicalizzazione e programmi di de- radicalizzazione. p.5 (https://journals.openedition.org/qds/1792) 
tutto sull'analisi delle relazioni e sulle influenze che quest'ultime possono avere nelle scelte degli individui; nello specifico, esamina la radicalizzazione unita al concetto di conflitto e dinamiche all'interno dei gruppi. Nello specifico, questa scuola di pensiero identifica degli elementi base per la comprensione del fenomeno:

- la "group polarization", cioè la propensione a portare il pensiero del gruppo ad una versione estremizzata;

- il "multiplier", cioè l'istinto del gruppo a diventare più unito in caso di minaccia;

- "l'outbidding", cioè l'attrattività che hanno i gruppi più radicali, considerati più devoti, messi in relazione con altri gruppi;

- la "condensation", in questo caso la volontà dello Stato di combattere i suddetti gruppi porta ai membri più deboli a lasciar perdere la causa, creando un gruppo con soli membri decisi e radicalizzati;

- il "fissioning", inteso come la possibile nascita di contrasti all'interno del gruppo, i quali portano alla scissione di quest'ultimo in più nuclei, in contrasto tra loro, che lottano per identificarsi come il più estremo dell'altro. ${ }^{28}$

La terza corrente di pensiero sociologico, la Conversion Theory, invece abbandona lo studio sul gruppo e la collettività e si concentra sulla sfera individuale in relazione ai cambiamenti di ideologie e credenze che un soggetto può compiere. Secondo la suddetta scuola di pensiero la "conversione" avviene in seguito ad una combinazione di eventi negativi, come ad esempio il non riuscire a raggiungere il proprio obiettivo di vita. Esistono cinque tipi di conversione:

- il mystical, un tipo di conversione che si manifesta in maniera improvvisa in seguito ad un evento traumatizzante;

- l'experimental, in questo caso la conversione avviene per sentirsi parte integrante del gruppo;

- l'affectional, la conversione è frutto di un attaccamento verso gli altri;

- il revivalist, frutto di un'esperienza nel contesto di folla;

- il coercive, nasce da una forma di coercizione psicologica in cui l'individuo che si è sempre trovato isolato rispetto alla società, si ritrova a subire influenze da parte di gruppi.

Risulta chiaro come, le conversioni sopra descritte, siano attribuibili ai processi di radicalizzazione. ${ }^{29}$

Come già anticipato, anche gli studi criminologici hanno cercato di dare una spiegazione al fenomeno della radicalizzazione partendo dai fattori macro-sociali. Diverse sono le teorie a cui il filone ha fatto riferimento, dalla "teoria del controllo sociale", alle "teorie della criminologia critica" fino ad arrivare "alla teoria generale della tensione".

La prima teoria prende in considerazione il concetto di "conformismo". Secondo Hirschi, autore della teoria, esiste una naturale propensione alla devianza e di conseguenza a dover essere analizzato è il livello di adesione al conformismo: un individuo è più portato a deviare quando il legame con la società e con le istituzioni è più debole. Plasmando la teoria in relazione alla radicalizzazione islamica, l'individuo è più incline ad unirsi alle cellule terroristiche quando non è inserito in una rete di relazioni, ma al contrario è segnato da un distanziamento culturale e relazionale. ${ }^{30}$

Per quanto riguarda le teorie della criminologia critica, partono dall'idea che la radicalizzazione sia frutto della violenza politica e delle caratteristiche delle odierne "società a rischio"; all'interno di esse le "interconnessioni delle azioni e reazioni di Stato nella produzione della violenza" porterebbero alla criminalità. ${ }^{31}$

Infine, risulta estremamente interessante la teoria generale della tensione di Agnew applicata al processo di radicalizzazione islamica. La suddetta teoria trova le sue radici dal concetto di anomia di Durkheim, intesa come assenza di leggi che regolano il comportamento umano e di Merton, il quale con il concetto di anomia intende il divario tra le mete culturali e la mancanza di adeguati mezzi istituzionalizzati per poterle raggiungere.

Agnew, sostiene che la tensione (dovuta alla frustrazione per non avere l'accesso ai mezzi per poter raggiungere la meta) porti ad emozioni negati che a loro volta portano a considerare un'azione deviante risolutiva. L'autore analizza come la tensione si pone alla base della radicalizzazione e il perché non tutti i soggetti che vivono uno stato di frustrazione decidono di unirsi al terrorismo.

Quando si parla di terrorismo, si parla di "tensione collettiva", la quale porta a generare un'azione violenta. La suddetta, si manifesta quando si presentano tre condizioni:

- è una tensione di elevata intensità; conseguenza di un'azione che ha procurato un danno e viene perpetuata per un lungo periodo.

- vige un sentimento di ingiustizia, che nasce quando si attuano azioni contrarie ai valori collettivi condivisi (anche quando non sono necessarie per il raggiungimento degli obiettivi).

- la tensione viene generata da altri attori considerati più potenti, come ad esempio gli attori statali.

I tre punti possono essere riportati nel contesto islamico: i cittadini musulmani hanno subito danni per lungo periodo, subiscono ingiustizie poiché sono una minoranza nel contesto sociale e politico e allo stesso tempo, si sentono minacciati dalla presenza dell'Occidente. Contesto che porta a generare la sopracitata "tensione", aumentando la probabilità di atti terroristici. Il passaggio dallo stato di tensione all'azione vera e propria si manifesta quando, l'individuo si distacca dalla società e si unisce in una comunità di simili, alienandosi dal contesto circostante e creando un'identità collettiva. ${ }^{32}$

Non tutti i soggetti che vivono uno stato di tensione, però, finiscono per compiere azioni devianti. Questo perché, bisogna analizzare le "modalità personali di reazione emotiva alle tensioni", cioè la "disponibilità" verso il compiere un'azione violenta. Principalmente, il motivo che differenzia coloro che decidono di generare terrore da coloro che scelgono una via alternativa, è legata alla possibilità di utilizzare o meno mezzi legittimi. ${ }^{33}$

Dalle diverse teorie, si può constatare che il fenomeno non riguarda solo le classi sociali poste ai margini della società o persone con scarso grado di istruzione, ma comprende diverse componenti: dai musulmani europei di seconda generazione, a coloro che decidono di convertirsi, in maniera anche repentina per potersi avvicinare all'ideologia estremista, persone che hanno subito traumi e così via.

Di seguito, un elenco che evidenzia i diversi tratti caratteristici del terrorista, andando a definire quello che può essere chiamato il "profilo criminologico del terrorista": 34

Tratti demografici:

- Età compresa dai 15 ai 34 anni

- Principalmente maschi (occasionalmente donne)

\section{Ibidem.}

29 Ivi, pag. 7.

30 Black D. (2004), The Geometry of Terrorism, in M. Deflem (a cura di), Terrorism and Counter-Terrorism: Criminological Perspectives, London, Elsevier. p.18

31 Crocitti, radicalizzazione islamica e marginalità, una lettura criminologica; Carocci Editori,2020. p.50

32 Agnew R., A General Strain Theory of Terrorism, in "Theoretical Criminology", 2010

33 Ivi, p. 144/146.

34 Mastronardi, Palermo, il profilo criminologico: dalla scena del crimine ai profili socio-psicologici; Giuffrè editore, Milano, 2005 pp.341-342 
- Tasso d'istruzione medio/basso

- Livello socio-economico generalmente basso

- Contatto con gruppi violenti

- Concetto di violenza come stile di vita

- Tratti di Personalità:

- Arrogante

- Sicuro di sé

- Sospettoso

- Freddo/calcolatore

- Litigioso (anche se spesso forzatamente contenuto)

- Paranoide

- Dedicato totalmente alla propria ideologia

- Privo di rimorso

- Capacità di disumanizzare l'essere umano

- Non curante della sorte delle sue vittime

- Comportamento apparentemente civile e non sospettoso

- A volte possiede alto Quoziente Intellettivo e modi tendenzialmente raffinati.

Esistono, inoltre, manuali del jihad, dove all'interno vengono spiegati tutti i comportamenti che un terrorista deve attuare nelle diverse situazioni. All'interno sono elencati anche i requisiti per poter essere selezionati dal jihadismo (permettendo agli esperti di delineare con più precisione alcune caratteristiche del terrorista) che comprendono l'essere astuto, intelligente, calmo, capace di resistere all'arresto e alla prigione, saper accantonare le proprie tradizioni e i precetti musulmani per ingannare il nemico e saper affrontare il trauma dopo aver compiuto delle stragi.

In conclusione, il terrorista è un soggetto convinto di essere superiore agli altri, di essere il predestinato a dover porre la parola fine alla lotta tra il bene e il male e, per questo, considera le proprie vittime come soggetti indesiderati. ${ }^{35}$

\section{Conoscere l'Islam per contrastare il radicalismo}

Ad oggi, il fenomeno della radicalizzazione è divenuto uno degli argomenti principalmente trattati dagli esperti della sicurezza, discusso sia a livello nazionale, europeo che internazionale. Il motivo di interesse su diversi livelli è principalmente dovuto alla sua trasformazione da minaccia "esogena" ad "endogena"; se prima il terrorismo poteva essere circoscritto in quei territori esteri, dove le organizzazioni erano ormai radicate, adesso il nemico agisce anche nel resto del mondo.

\section{Radicalizzazione in carcere: Islam e "best practices" in carcere}

Dai diversi studi effettuati sui processi di radicalizzazione, si è potuto constatare come siano nati nuovi percorsi che vanno a differenziarsi dal classico dualismo tra recluta e reclutato. Ad oggi, esistono percorsi di radicalizzazione "autonomi", i quali possono avvenire attraverso internet o all'interno delle carceri. In quest'ultimo caso, risulta evidente come le interconnessioni sociali e l'aver condiviso dei vissuti simili, possano incidere sulla scelta di un individuo ad avvicinarsi al terrorismo. In questo contesto, la scoperta o riscoperta della religione, può portare per alcuni soggetti, a vivere diversamente la detenzione (soprattutto per i detenuti stranieri che si ritrovano a non avere nessun legame con l'ambiente circostante).

Il carcere è diventato per il jihadismo terreno fertile per poter ricercare nuove reclute; Inghilterra, Francia e, in misura minore l'Italia, si ritrovano ad affrontare questa nuova sfida.

Difatti, proprio nei Paesi sopracitati sono state effettuate delle indagini all'interno di istituti penitenziari, le quali hanno portato alla luce due fenomeni: il primo riguarda la radicalizzazione di criminali comuni, non legati al mondo del radicalismo, che pur non manifestando nessun avvicinamento religioso nel momento in cui sono arrivati in carcere, hanno subìto influenze da altri detenuti. Il secondo fenomeno è legato alla questione riguardante l'imposizione della religione islamica da parte di alcuni detenuti; difatti, i "non musulmani" spesso subiscono una costrizione violenta verso una conversione forzata che comprende tutti i rituali annessi alla religione stessa, inclusi i divieti alimentari. ${ }^{36}$

L'episodio esplicativo, che ha reso noto all'opinione pubblica la minaccia della radicalizzazione in carcere, è avvenuto in Francia. Michael Chiolo, un detenuto che ha subito un processo di radicalizzazione all'interno delle stesse mura carcerarie, ha ferito due agenti penitenziari per vendicare l'uccisione di Chekat, responsabile dell'attentato a Strasburgo nel 2018 ed ex compagno di carcere di Chiolo. ${ }^{37}$.

In Francia, già nel 2008, si registrava all'interno degli istituti penitenziari la presenza di 144 terroristi. ${ }^{38}$ In Spagna, i servizi segreti sono riusciti a decodificare un linguaggio in codice utilizzato dai gruppi estremisti per mantenere un canale di comunicazione con l'esterno.

In Italia, secondo la Relazione del Ministero della Giustizia ${ }^{39}$, le statistiche del 2018 contato 7.169 detenuti di origine musulmana e praticanti; di questi: 97 ricoprono il ruolo dell'imam, altri 88 hanno deciso di ricoprire il ruolo del "promotore", cioè portavoce delle istanze degli altri detenuti nei confronti della direzione penitenziaria, mentre son state 44 le conversioni avvenute durante la detenzione.

Il fenomeno in Italia è sicuramente di intensità minore rispetto all'Europa, ma è da tenere sicuramente sotto osservazione, vista la minaccia sempre più realistica: Anis Amri, autore dell'attentato di Berlino del 2016, si è avvicinato al jihadismo in un carcere siciliano e la sua condanna non era legata ad alcuna azione estremista. Le statistiche del 2020 riportano invece la presenza di 66 imputati e/o condannati per reati legati al terrorismo internazionale di matrice islamica. I condannati sono stati inseriti all'interno dei circuiti di "Alta Sicurezza", istituiti in Italia nel 2009 e dedicati interamente ai soggetti imputati o condannati per reati di terrorismo. ${ }^{40}$

Per rimarginare il fenomeno in espansione, i Paesi maggiormente colpiti hanno capito che la risposta deve essere immediata ed efficace. Fondamentale e strettamente necessaria al fine di bloc-

35 Ivi, p. 344.

36 La radicalizzazione del terrorismo islamico, elementi per uno studio del fenomeno di proselitismo in carcere, Istituto Superiore di Studi Penitenziari, 2012. p.32-33 https://www.giustizia.it/resources/cms/documents/radicalizzazione_del_terrorismo_islamico.pdf)

37 Marone F., La radicalizzazione jihadista in carcere: un rischio anche per l'Italia. $2019 \mathrm{https} / / \mathrm{www}$.ispionline.it/it/pubblicazione/la-radicalizzazione-jihadista-carcere-un-rischio-anche-litalia-22475

38 La radicalizzazione del terrorismo islamico, elementi per uno studio del fenomeno di proselitismo in carcere, Istituto Superiore di Studi Penitenziari, 2012. p.32-33

39 Relazione del Ministero sull'amministrazione della giustizia anno 2017, Inaugurazione dell'Anno Giudiziario 2018 - Dipartimento dell'amministrazione penitenziaria, gennaio 2018, p. 29. https://www.giustizia.it/ resources/cms/documents/anno_giudiziario_2018_dap.pdf

40 Marone F., La radicalizzazione jihadista in carcere: un rischio anche per l'Italia. 2019 https://www.ispionline.it/it/pubblicazione/la-radicalizzazione-jihadista-carcere-un-rischio-anche-litalia- 22475 
care l'avanzare del jihadismo in carcere è la formazione degli operatori carcerari, degli educatori e, infine, degli assistenti sociali; essi devono essere capaci di valutare il "risk assessment" della radicalizzazione e devono avere "la conoscenza" di ogni detenuto.

Il modello italiano ha basato la sua risposta su misure di vigilanza, sorveglianza, osservazione e controllo preventivo al fine di portare avanti l'obiettivo della detenzione, definito dall' art.27 della Costituzione ${ }^{41}$ e dall'Ordinamento giudiziario. Il monitoraggio dei detenuti a rischio radicalizzazione si attua attraverso un'analisi su diversi livelli:

- Primo livello-alto; i soggetti presi in osservazione sono già stati condannati per reati legati al terrorismo o hanno assunto atteggiamenti tendenzialmente radicali.

- Secondo livello- medio; in questo livello sono inseriti quei detenuti che hanno assunto determinati atteggiamenti che possono far presupporre un avvicinamento alle idee radicali.

- Terzo livello- basso; sono inseriti in questo gruppo quei detenuti che non fanno più parte dei livelli precedenti, ma che necessitano comunque un'osservazione. ${ }^{42}$ Il modello francese ha evidenziato tre livelli di allarme:

- Livello giallo; il segnale di allarme è dato dalla presenza nelle celle di materiale connesso al jihadismo, dalla formazione di gruppi chiusi ed eventuali pressioni sugli altri detenuti.

- Livello arancione; il segnale di allarme è dato dal nascere di preghiere collettive con un imam autoproclamato e da sfide riposte alle autorità penitenziarie.

- Livello di allarme rosso; in questo livello si registrano aggressioni nei confronti di altri detenuti o degli agenti, oppure si possono notare nascite di sommosse o azioni distruttive. ${ }^{43}$

Anche il Consiglio dell'Unione Europea ha riconosciuto la radicalizzazione in carcere come una minaccia reale, dando un contributo per contrastarlo. La presidenza ha inviato un questionario agli Stati Membri, per raccogliere dei dati inerenti alle diverse politiche di prevenzione attuate, in modo da poter avere una raccolta e definire delle linee guida di "buone pratiche di contrasto", ${ }_{4}$

\section{Buone pratiche di prevenzione e contrasto al terrorismo internazionale}

È soltanto dopo aver affrontato il fenomeno del terrorismo transnazionale in ogni sua sfaccettatura che si può introdurre il concetto di "buone pratiche" di contrasto al terrorismo stesso. L'obiettivo di questo paragrafo sarà quello di fornire una serie di procedure utili sia per limitare le attività dei nuclei estremisti già attivi, sia per evitare l'avvicinamento di nuove reclute verso tale fenomeno al fine di prevenire, contrastare e controllare la minaccia.

La strategia, deve basarsi su fondamentali quattro pilastri:

- Risposta

- Perseguimento

- Protezione

- Prevenzione

\section{Risposta}

Ad avvenuto attentato le azioni da compiere devono essere estremamente efficaci sia per attenuare gli effetti che l'evento comporta sui cittadini, sia per eliminare la possibilità che tale minaccia possa rimanifestarsi. La risposta deve avvalersi di un coordinamento tra tutti i mezzi di comunicazione e supporto disponibili; in quest'ottica ha agito anche l'UE offrendo supporto nel caso in cui uno Stato non abbia sufficienti risorse disponibili per affrontare l'emergenza. ${ }^{45}$.

Le azioni principali in questa fase sono tre: stabilire le modalità di coordinamento dell'Unione Europea per la gestione dell'emergenza, puntare sulla cooperazione internazionale e creare una rete di supporto per le vittime del terrorismo e le rispettive famiglie.

Gli strumenti per perseguire l'obiettivo di una risposta efficace possono essere svariati. Una solida collaborazione internazionale, europea e nazionale può essere un ottimo punto di partenza perseverando sull'utilizzo di canali comunicativi celeri allo scopo di avere uno scambio continuo di informazioni. Il COSI, ${ }^{46}$ infatti, ha evidenziato la necessità di rafforzare la cooperazione tra Ue, Turchia, i Paesi del Nord Africa, il Medio Oriente e i Balcani occidentali. ${ }^{47}$

A livello operativo, per puntare sulla buona riuscita di una risposta efficace i primi passi da compiere sono: l'analisi del fenomeno e dei rischi; analizzare il percorso dalla nascita all'evoluzione del terrorismo, creare un profilo storico, individuale e di gruppo e capire le cause che portano al compimento da parte di tali soggetti di determinate azioni violente, sono le prime operazioni da compiere al fine di attivare iniziative investigative più efficaci. Successivamente, anche lo studio della documentazione e della propaganda attuata dai diversi gruppi terroristici può risultare utile allo scopo di comprendere l'ideologia di base che ha portato al compimento di determinate azioni. Infine, attuare un'analisi politico- ideologica, psicologia, sociologica e criminologica dei militanti "conosciuti" può aiutare a creare un profilo generico del terrorista al fine di rendere più agevole la ricerca di tali soggetti.

Sempre a livello operativo, un'altra operazione utilizzare può risultare la tecnica del "doppio binario": dare la possibilità al terrorista catturato di pentirsi o dissociarsi. Nel primo caso il terrorista si pente di ciò che ha fatto e dà informazioni al fine di bloccare altri attentati, di conseguenza rinuncia all'azione armata, attua un auto critica e inizia il processo di liberazione attraverso la collaborazione; la seconda opzione non obbliga il terrorista a rivelare informazioni utili alle indagini, ma gli impone di dissociarsi pubblicamente dalla propria esperienza politica e operativa, denunciandone l'inefficacia. In questo modo, si punta a colpire sulla serietà e credibilità dell'organizzazione terroristica, disincentivando le possibili nuove reclute.

41 La responsabilità penale è personale. L'imputato non è considerato colpevole sino alla condanna definitiva. Le pene non possono consistere in trattamenti contrari al senso di umanità e devono tendere alla rieducazione del condannato.

42 Paterniti C, numero e strategie di contrasto. Il difficile bilanciamento tra sicurezza e diritti. http://www.antigone.it/quindicesimo-rapporto-sulle-condizioni-di-detenzione/radicalizzazione/\#1557585052619. baefc846-9921

43 La radicalizzazione del terrorismo islamico, elementi per uno studio del fenomeno di proselitismo in carcere, Istituto Superiore di Studi Penitenziari, 2012. p.35-36 https:/www.giustizia.it/resources/cms/documents/radicalizzazione_del_terrorismo_islamico.pdf

44 Consiglio dell’Unione Europea, Bruxelles 2019. https://data.consilium.europa.eu/doc/document/ST-9366-2019-INIT/it/pdf

45 Dolgetta D., Europa e terrorismo. La strategia dell'Unione Europea per contrastare il terrorismo internazionale. Università degli studi di Salerno, 2017. P.13. http://www.centrostudieuropei.it/jeanmonnet/wpcontent/uploads/2015/04/Bianca-Dolgetta_Europa-e-terrorismo.pdf

46 Comitato permanente per la Cooperazione Operativa in materia di Sicurezza Interna

47 Gelao N. e collaboratori, la condivisione dell'intelligence per finalità di prevenzione e contrasto del terrorismo internazionale nell'Unione Europea. Una cooperazione ulteriormente rafforzata è utile e possibile? 2016. p.77 https://www.difesa.it/SMD_CASD/IM/CeMiSS/DocumentiVis/ Rcerche_da_pubblicare/pubblicate_nel_2018/Ricerca_AL_S_09.pdf 


\section{Perseguimento}

Con perseguimento si intente arrivare a colpire i terroristi non solo sul territorio occidentale, ma anche oltre frontiera. Lo scopo è quello di smantellare le diverse reti di reclutamento, seguire le diverse piste finanziare ed eliminarle la possibilità di reperire $\mathrm{i}$ materiali necessari per compiere i propri attacchi. ${ }^{48}$

Anche in questo caso ci vuole un impegno da parte di tutti gli attori coinvolti nella lotta al terrorismo per cercare di ostacolare l'accesso alle armi e agli esplosivi da parte dei terroristi e ostacolare il finanziamento al terrorismo. ${ }^{49}$

\section{Protezione}

Parlare di protezione significa parlare della difesa del proprio territorio. In questo caso le azioni devono essere rivolte al rafforzamento della sicurezza delle frontiere e migliorare i controlli riguardante i trasporti. L'agenzia europea per la gestione delle frontiere esterne, Frontex, sotto questo aspetto ricopre un ruolo fondamentale e, un'effettiva analisi del rischio delle frontiere da parte di questa, potrebbe essere un valido strumento di contrasto. ${ }^{50}$

Il controllo dei flussi migratori e delle frontiere, la collaborazione di tutti gli Stati Membri nello scambio di informazioni e l'utilizzo della "policy cycle" sono le operazioni alla base di questo pilastro. ${ }^{51}$

Con policy cycle si intende "la suddivisione del processo di policy in varie fasi e sottofasi, in modo tale da poter facilitare il più possibile la comprensione del processo di definizione delle politiche pubbliche, ovvero capire più di preciso il ruolo degli attori coinvolti all'interno di tale processo e gli interessi da loro perseguiti. il modello più semplice di Policy Cycle si articola in cinque fasi: la definizione dell'agenda, formulazione della politica, la fase decisionale, la messa in opera della politica e la valutazione." 52

A livello europeo è importante puntare ad una buona policy che non venga considerata né uno stato né una somma di stati, ma una politica d'integrazione con delle forti basi. Ci si potrebbe rifare alla teoria di Fabbrini, che ipotizza una policy europea non sulla base dei modelli decisionali, ma sulla base della partecipazione: "dal basso" (decisioni intergovernative) e "alto" (politica estera e difesa in chiave sovranazionale). I due livelli non andrebbero contrapposti né fusi, ma collegati tra loro. ${ }^{53}$

\section{Prevenzione}

Per prevenire i processi di radicalizzazione, l'impegno deve esserci non solo da parte dell'Ue, ma anche a livello regionale e locale. Con la globalizzazione che ha favorito la facilitazione degli spostamenti di individui, persone, denaro e ha facilitato la comunicazione, ogni singolo individuo può avvicinarsi all'ideologia estremista.

Per questo motivo di estrema importanza risulta essere l'attuazione di una strategia riguardante la comunicazione; la suddetta risulta essere un'arma sia a nostro vantaggio che svantaggio: con la nascita di internet i terroristi hanno trovato sia un canale celere per attuare la propria propaganda in tutto il mondo allo scopo di reclutare nuovi militanti, sia un'agevolazione nel coordinamento delle reti terroristiche. ${ }^{54}$

Allo stesso tempo, però, il medesimo strumento è anche nelle mani dell'Occidente attraverso il quale può seguire le diverse tracce digitali per arrivare ai terroristi, come ad esempio il movimento di denaro o intercettazioni di messaggi estremisti.

La comunicazione risulta di estrema importanza anche ai fini dell'integrazione, elemento indispensabile per una buona prevenzione al fenomeno del terrorismo internazionale. Creare luoghi comuni per le diverse culture, avvicinarsi al mondo islamico senza pregiudizi, provare a conoscere la loro religione ed integrarli nella società diminuirebbe di molto la propensione di determinati soggetti al processo di radicalizzazione.

Agire sulle seconde generazioni, è un altro aspetto di questo pilastro di estrema importanza. Quando parliamo di "seconde generazioni" facciamo riferimento a quei ragazzi nati da genitori stranieri in un luogo diverso rispetto la loro provenienza. Molto spesso, essi, si ritrovano a combattere una crisi d'identità: non si sentono pienamente ancorati alle radici dei genitori e, molto spesso, non si sentono parte del mondo in cui si trovano, costretti a subire discriminazioni e trattamenti diversi e alle volte, la propaganda estremista, riesce a far trovare una dimensione a questi soggetti. ${ }^{55}$

Una buona pratica di contrasto prevede la totale integrazione di coloro che non si sentono parte della società, attraverso campagne di sensibilizzazione nelle scuole $o$ attraverso attività di inclusione sociale per le comunità islamiche presenti sul territorio. Diminuire quei quartieri esclusivamente musulmani porterebbe diminuire quel fenomeno di "ghettizzazione" e allo stesso tempo potrebbe generare un controllo sociale spontaneo. Il meccanismo del controllo sociale è costituito dall'insieme di soggetti a cui spetta istituzionalmente la difesa delle norme di comportamento sociale e la salvaguardia delle regole della convivenza da possibili violazioni. Il controllo sociale spontaneo nasce per il fatto stesso che le persone hanno interiorizzato i comportamenti più consoni e distinguono quelli che non lo sono. Nel momento in cui osservano comportamenti di natura deviante, nonostante non siano le autorità competenti, possono denunciare il fatto e renderlo noto

Ai fini della prevenzione, può risultare utile studiare i "tempi di attivazione". Con tale espressione viene definito il tempo che intercorre tra il primissimo contatto con l'ideologia estremista e la prima azione violenta compiuta, la quale stabilisce l'adesione al pensiero jihadista. Gli esperti devono essere consapevoli che $\mathrm{i}$ tempi di attivazione sono diversi a seconda della tipologia di radicalizzazione. Ad esempio, in quella cosiddetta "faccia a faccia", il primo approccio è da considerarsi nell'incontro con la figura centrale ${ }^{56}$ del processo di radicalizzazione mentre, invece, nell'autoradicalizzazione il primo passo risulta essere l'utilizzo consistente di materiale jihadista. Per quanto riguarda invece "l'azione", può essere intesa sia come la realizzazione di un attentato ma anche la volontà di partire verso i territori occupati dalle organizzazioni terroristiche. Alcuni studiosi hanno misurato in anni il tempo di atti-

48 Dolgetta D., Europa e terrorismo. La strategia dell'Unione Europea per contrastare il terrorismo internazionale. 2017. p.12. http://www.centrostudieuropei.it/jeanmonnet/wp-content/uploads/2015/04/ Bianca-Dolgetta_Europa-e-terrorismo.pdf

49 Per un maggior approfondimento in merito alla lotta al finanziamento al terrorismo: https://www.consilium.europa.eu/it/policies/fightagainst-terrorism/fight-against-terrorist-financing/ 20.11.2020

50 Ivi, p. 11

51 Gelao N. e collaboratori, la condivisione dell'intelligence per finalità di prevenzione e contrasto del terrorismo internazionale nell'Unione Europea. Una cooperazione ulteriormente rafforzata è utile e possibile? 2016. p.77 https://www.difesa.it/SMD_CASD/IM/CeMiSS/DocumentiVis/ Rcerche_da_pubblicare/pubblicate_nel_2018/Ricerca_AL_S_09.pdf

52 Ibidem.

53 Sbailò, crisi nordafricana Katastrophè e occasione di rilancio per l'Europa, federalismi 2019. p.29

54 https://www.difesa.it/SMD/CASD/IM/CeMiSS/DocumentiVis /Rcerche_da_pubblicare/pubblicate_nel_2018/Ricerca_AL_S_09.pdf. 20.11.20

55 http://www.integrazionemigranti.gov.it/archiviodocumenti/minorie-g2/Documents/INSIDE $\% 20$ OUT $\% 201 \% 2$ IItalia $\% 20$ sono $\% 20$ anch $\%$ 27io.pdf 20.11.2020

56 Il reclutatore. 
vazione: varia da meno di un anno a quindici anni; dagli studi effettuati, il secondo caso risulta meno realistico, mentre molto più frequente è il primo dato. In linea generale, il passaggio dall'avvicinamento all'ideologia estremista all'azione varia da un anno ai quattro. ${ }^{57}$ Informazioni importanti, perché è in questo lasso di tempo che si deve agire, cercando di dissuadere il soggetto a passare all'atto pratico.

Al netto di ciò risulta fondamentale avere una panoramica completa a breve, medio e lungo termine delle strategie delle organizzazioni terroristiche. Non è da trascurare il fatto che i terroristi sono molto informati e aggiornati sulle strategie e nuove metodologie che il mondo occidentale mette in atto per contrastarli; questo consente loro di poter agire con determinate precauzioni, passando indisturbati. Una metodologia utile può essere " 1 'analisi previsionale mutuato dalla teoria dei giochi con equilibri dinamici".

In quest'analisi si prende in considerazione l'azione della controparte (A) in un breve arco temporale $(\mathrm{t})$, valutando a priori le probabili reazioni $(\mathrm{R})$ successive a questo arco di tempo $(\mathrm{t}+\mathrm{l})$ dei soggetti di risposta (gli organi preposti all'antiterrorismo)

$$
A=f(\operatorname{Pr}(R) t+1) t
$$

Questo schema ci permette di capire che le reazioni provocate da un attacco terroristico (paura, incertezza ect) sono prevedibili, considerate dal nemico come un obiettivo e soprattutto condizionabili. Parlando di uno schema interattivo, vuol dire che ogni attore in gioco può condizionarne la riuscita ${ }^{58}$.

È da questo punto che si deve partire.

Il terrorismo resta un nemico difficile da combattere, capace nel tempo di adattarsi alle circostanze e di infiltrarsi in Occidente. Lo Stato democratico in opposizione alle organizzazioni terroristiche deve continuare ad affermare i principi fondamentali e i valori sul quale si è sempre basato. Dopo l'attentato dell'11 settembre 2001 il senso di insicurezza è aumentato sempre più nei cittadini e di risposta gli Stati hanno attivato nuove misure repressive, dagli inasprimenti delle vecchie pene all'inserimento di nuove fattispecie di reato.

Il punto cruciale è definire la sottile linea entro le quali le nuove misure possono essere considerate legittime senza andar a ledere le libertà individuali. ${ }^{59}$

La vera sfida dell'Occidente è trovare un punto d'incontro tra la "tolleranza" e la "sorveglianza".

Ogni singolo Stato deve puntare a sradicare le idee estremiste senza allontanare le persone, avvicinandosi alle comunità islamiche non per osteggiarle, ma per combattere insieme la minaccia terroristica. Bisogna entrare nell'ottica nella quale gli estremisti sono solo una piccola parte deviata di un gruppo molto più ampio di musulmani che, in primis, considerano le loro azioni totalmente sbagliate. Ben oltre il 90 per cento dei musulmani è estrano alle logiche del terrorismo ed è a questa grande percentuale che bisogna chiedere di attivarsi per rendere più proficua la lotta contro il terrorismo e soprattutto la radicalizzazione, divenendo nuovi occhi e nuove orecchie per segnalare possibili nuove reclute al mondo del terrorismo. ${ }^{60}$.

Ciò può avvenire affiancando i due mondi senza dover rimarcare le differenze, applicando allo stesso tempo il concetto di tolleranza e sorveglianza.

È constatazione comune che il terrorismo di oggi è strutturalmente e operativamente diverso dalle forme precedentemente avute. Sicuramente, creare e attuare una strategia efficace per contrastare il terrorismo internazionale è uno degli obiettivi da perseguire, ma alla luce dell'evoluzione dell'organizzazione del terrorismo, non si può trascurare la nascita di una componente del fenomeno sempre più individualista. Esso si muove al di là dei propri confini e per questo, essendo la natura della minaccia "asimmetrica" non basta agire solo sul piano operativo svolto dall'intelligence, o attraverso politiche penali nazionali, ma risulta fondamentale disporre azioni volte alla prevenzione sociale. Tale obiettivo può essere perseguito, appunto, incentivando il dialogo e l'integrazione tra le culture e le religioni.

Conoscere l'islam è il primo importante passo per diminuire il distanziamento sociale. La creazione di un "islam europeo" (come i già esistenti islam di cultura turca o indiana), potrebbe aiutare alla causa, creando istituti in cui i musulmani possano trovare intermediari e dialogare, sentirsi parte di qualcosa.

"Il terrorismo nuoce in primo luogo proprio ai musulmani, e per questo crediamo che uno dei modi migliori per contrastarlo sia quello di stabilire solidi rapporti con le comunità islamiche, che possono dare un contributo decisivo nell'individuare ed emarginare il fenomeno". ${ }^{61}$

\section{Bibliografia}

Agnew R., A General Strain Theory of Terrorism, in "Theoretical Criminology", 2010.

Alcaro R., Documentazione per le delegazioni presso le Assemblee internazionali: la lotta al terrorismo dopo l'11 settembre; principali iniziative degli USA E UE. 2005

Allan, H.; Glazzard A., Jesperson, S., Reddy-Tumu, S., Winterbotham E., Drivers of violent extremism, hypotheses and literature review, Royal United Service Institute (RUSI), 2015.

Anastasio N., Il dilemma della sicurezza in Iraq: vecchie e nuove minacce. Amistades, 2020.

Anastasio N., Il risveglio dello Stato Islamico in Iraq. Istituto Analisi Relazioni Internazionali, 2020.

Barile P. Il soggetto privato nella costituzione italiana, Padova, 1953.

Bassu C., La legislazione antiterrorismo e la limitazione della libertà personale in Canada e negli Stati Uniti,2006.

Bellotto A., Al Qaeda tra perdita di leadership e sfide dei gruppi affiliati. Insideover, 2020.

Berna A., Cos'è la jihad e perché è importante capirne il suo significato, 2016.

Black D. (2004), The Geometry of Terrorism, in M. Deflem (a cura di), Terrorism and Counter-Terrorism: Criminological Perspectives, London, Elsevier.

Borum, Radicalization into Violent Extremism I: A Review of Social Science Theories, 2011.

Brynjar Lia, Architect of Global Jihad: The Life of Al-Qaida Strategist Abu Mus'ab AlSuri, New York, Columbia University Press, 2008.

57 Pettinari, radicalizzazione jihadista in Europa. Indagine sul "tempo di attivazione" degli individui radicalizzati. 2019 https://www.difesa it/SMD_CASD/IM/CeMiSS/DocumentiVis/Osservatorio_Strategico_2019/ OS_03_2019/ITA/24_PETTINARI_SL_03_2019.pdf

58 Pollari N, Un nuovo approccio all' analisi del fenomeno del terrorismo internazionale. Il Politico, vol. 72, No. 3 (216), 2007.

59 Feola A., la lotta al terrorismo: misure di contrasto in ambito nazionale ed internazionale. p. $58 \mathrm{https} / / /$ www.difesa.it/SMD_/CASD/ IM/CeMiSS/Pubblicazioni/ricerche/Documents/Ricerche_2013/Söciologia _Militare/CeMiSS_Ricerca_Terrorismo_Feola.pdf

60 https://www.redattoresociale.it/article/notiziario/bruxelles i terroristi_di_seconda_generazione_frutto_delle_politiche_europee_20.11.20

${ }^{6} 1^{-} \bar{C}_{\text {Citazione di Alberto }}^{-}$Ventura, direttore $\overline{\text { del laboratorio sul }}$ Mediterraneo Islamico dell'università della Calabria. 
Caselli e Spataro, La magistratura italiana negli anni di piombo; 2009.

Ceremigna J, Regno Unito: arriva la nuova legge sul terrorismo. Sicurezza internazionale, 2010.

Cesta G., Radicalizzazione islamica e misure di prevenzione: dall'esperienza europea spunti per implementare il sistema di prevenzione italiano. SKAI- centro di studi Kore sul Costituzionalismo Arabo e Islamico, 2020.

Cole \& Dempsey, terrorism and the constitution: sacrificing civil liberties in the name of national; New Pr, 2002.

Cossiga A., Per i jihadisti il coronavirus è una punizione e una benedizione. Limes, 2020

Crocitti S., Radicalizzazione islamica e marginalità. Una lettura criminologica. Carocci editore, 2020.

Dacrema E., Il jihadismo ai tempi del coronavirus. Ispionline, 2020 .

David S. Hilzenrath e John Mintz, "More Assets on Hold in AntiTerror Effort; 39 Parties Added to List of Al Qaeda Supporters", Washington Post, 13 October 2001.

De Vivo J., Francia: Dallo stato di emergenza alla nuova legge antiterrorismo, 2018;

Delfino, La legislazione antiterrorismo in Francia e in Russia. Un difficile equilibrio tra sicurezza dei cittadini e diritti, 2019.

Denaro R., Sillabo: conoscere l'islam per contrastare il radicalismo. Università Orientale di Napoli, 2018.

Digos, Analisi della perquisizione di viale Jenner, 15 settembre 1997, e memorandum della Digos sul Centro, 20 maggio 1994.

Dolgetta D., Europa e terrorismo. La strategia dell'Unione Europea per contrastare il terrorismo internazionale, Università degli studi di Salerno, 2017.

Fenucci, Sicurezza e diritti fondamentali del Regno Unito, 2012.

Feola A., La lotta al terrorismo: misure di contrasto in ambito nazionale ed internazionale.

Gelao N., e collaboratori, La condivisione dell'intelligence per finalità di prevenzione e contrasto del terrorismo internazionale nell'Unione Europea. Una cooperazione ulteriormente rafforzata è utile e possibile? 2016.

Giacalone G., i terroristi possono sfruttare il coronavirus? Insideover, 2020.

Giangrande A., Gesù Cristo vs Maometto e l'islamizzazione del mondo, 2016.

Gregory V., "Italia non ha terrorismo islamico interno, per oraAmato", Bloomberg News, 2007.

Guolo R., Il dibattito sulla radicalizzazione nelle scienze sociali. Guerini e Associati, Milano, 2017.

Halliday F., Nation and religion in the Middle East, Al Saqi, 2000.

Hilligsmann D., Stratégie de préntion du radicalisme violent en Communauté germanophone de Belgique 2016-2020.

Howlett M. e Ramesh M., Come studiare le politiche pubbliche. Il mulino, 2003

Il politico, vol. 72, No. 3 (216), il terrorismo internazionale analisi interdisciplinari. Un nuovo approccio all'analisi del fenomeno del terrorismo internazionale.

Il politico, vol.72, No. 3. (216). Il terrorismo internazionale e analisi interdisciplinari, 2007.

Istituto Superiore di Studi Penitenziari, La radicalizzazione del terrorismo islamico, elementi per uno studio del fenomeno di proselitismo in carcere, 2012.

Lamanna M., Il ruolo e il significato della comunicazione strategica nella galassia jihadista nel Medio Oriente contemporaneo. Centro Militare di studi strategici, 2016.

Laurano e Anzera, L'analisi sociologica del nuovo terrorismo tra dinamiche di radicalizzazione e programmi di de- radicalizzazione, 2017.
Laurenza P., Attentato suicida nel "triangolo della morte", 2020. Marco C., L'espansionismo jihadista dell'Is ("Stato Islamico") nell'area sub-sahariana e del sud-est pacifico, 2015.

Marone F., La radicalizzazione jihadista in carcere: un rischio anche per 1'Italia. Ispionline, 2019.

Mastronardi V, Leo S, Terroristi. Newton \& Compton 2005.

Mastronardi, Palermo, Il profilo criminologico: dalla scena del crimine ai profili socio-psicologici; Giuffrè editore, Milano, 2005

Mauceri C., L'11 settembre e le implicazioni. Notizie geopolitiche, 2020.

Mervin S, L'Islam. Fondamenti e dottrine, Bruno Mondadori, 2001.

Minerva Nasser-Eddine, Bridget Garnham, Katerina Agostino and Gilbert Caluya, Countering Violent Extremism (CVE) Literature Review, Canberra, Australian Government, Department of Defence, marzo 2011.

Munizio C., Nella mente del terrorista, l'ambizione di un passaggio alla storia. Le ragioni della follia; intervista al Presidente del Gruppo Italiano di Psichiatria (SIP)

Palladini G., Il terrorismo ai tempi del covid-19. Eurobull, 2020.

Paterniti C, Numero e strategie di contrasto. Il difficile bilanciamento tra sicurezza e diritti.

Pettinari, Radicalizzazione jihadista in Europa. Indagine sul "tempo di attivazione" degli individui radicalizzati. 2019

Presidenza del Consiglio dei Ministri, relazione sulla politica dell'informazione per la sicurezza, 2009, p. 19.

Pugliese M., La legge antiterrorismo in Francia: un bilancio due anni dopo. Ispionline 2019;

Reinares F., Jihadist Radicalization and the 2004 Madrid Bombing Network, CTC Sentinel vol. 2 Issue 11, Novembre 2009; Awan A., the 7/7 Attacks and a new Type of Terrorism, New Internationalist, 7 luglio 2015.

Relazione del Ministero sull'amministrazione della giustizia anno 2017, Inaugurazione dell'Anno Giudiziario 2018 Dipartimento dell'amministrazione penitenziaria, gennaio 2018.

Rover V, rischio sociale e sicurezza nazionale: il radicalismo islamico. Analisi della sua origine, teorizzazioni e possibili riflessi sulla sicurezza nazionale. Gnosis 2 .

Roy O., Generazione Isis: chi sono i giovani che scelgono il Califfato e perché combattono 1'Occidente. Milano, 2017

Sbailò C, la nuova sintassi del terrore. Gnosis 1/2005, 2005

Sbailò C., Carl Smith alla Casa Bianca. La dottrina Bush-Obama sullo stato di emergenza e i nuovi equilibri tra poteri negli Stati Uniti. 2010.

Sbailò C., Crisi nordafricana Katastrophè e occasione di rilancio per l'Europa, federalismi 2019.

Sbailò C., Terrorismo contemporaneo. Nuove minacce e vecchi diritti. Gnosis 3/2006, 2016.

Sipione L., Dal terrorismo politico alle nuove forme di terrorismo globale: strumenti di conoscenza e di contrasto in ambito nazionale ed europeo, 2017.

Tosini D., Sociologia dell'antiterrorismo: la struttura della lotta al terrorismo nelle democrazie liberali. Quaderni di sociologia, 2005

Tribunale di Milano Sentenza contro Mohamed Game e Hady Abdelaziz Mahmoud Adbel Kol, 10/1583, 4 ottobre 2010.

Vercelli C., Che cos'è la radicalizzazione. Note di riflessione a margine di un processo di politicizzazione di alcune minoranze islamiche.

Vidino V. Il jihadismo autoctono in Italia. Nascita, sviluppo e dinamiche di radicalizzazione. Istituto per gli Studi di Politica Internazionale, Milano, 2014 


\section{Sitografia}

http://www.integrazionemigranti.gov.it/archiviodocumenti/minori-e-g2/Documents/INSIDE\%20OUT\%201\%27Italia $\% 20$ sono $\% 20$ anch $\% 27$ io.pdf

http://www.interior.gob.es/documents/642012/5179146/PLAN+E STRAT\%C3\%89GICO+NACIONAL.pdf/d250d90e-99b5$4 \mathrm{ec} 9-99 \mathrm{a} 8-8 \mathrm{cf} 896 \mathrm{cb} 8 \mathrm{c} 2 \mathrm{f}$;

http://www.storiaxxisecolo.it/attaccoagliusa/precedenti.htm

http://www.studiperlapace.it/view_news_html?news_id=2005012 2123225

http://www.studiperlapace.it/view_news_html?news_id=2006081 6174647

http://www.unavox.it/ArtDiversi/DIV3347_Pecchioli_Mondo_liq uido.html

https://apnews.com/article/1bd95fe95acc9a052c1ca0bbcb3bb1a1

https://data.consilium.europa.eu/doc/document/ST-9366-2019INIT/it/pdf)

https://ec.europa.eu/home-affairs/what-we-do/networks/radicalisation_awareness_network/ran-and-member-states/repository_en

https://e-justice.europa.eu/content_european_arrest_warrant-90it.do

https:/espresso.repubblica.it/plus/articoli/2017/06/13/news/1islam-condanna-il-suicidio-e-questo-il-paradosso-del-terrorimo-jiadhista- 1.304208

https://radical.hypotheses.org/files/2016/04/EvaluationoftheNethe rlandscomprehensiveactionprogrammetocombatjihadism.pdf; https://rm.coe.int/16806ffla9;

https://tg24.sky.it/mondo/2017/11/01/attentati-new-york

https://www.beratungsstelleextremismus.at/

https://www.bmfsfj.de/blob/109002/5278d578ff8c59a19d4bef9fe $4 \mathrm{c} 034 \mathrm{~d} 8$ /strategie-der-bundesregierung-zur-extremismuspraevention-und-demokratiefoerderung-data.pdf;

https://www.camera.it/leg17/465?tema=prevenzione_dell_estremismo jahadista

https://www.camera.it/leg18/126?tab=\&leg=18\&idDocumento=2 $43 \&$ sede $=\&$ tipo $=$

https://www.centcom.mil/MEDIA/STATEMENTS/Statements-
View/Article/1891697/statement-from-us-central-commandon-strike-against-al-qaida-in-syria/

https://www.consilium.europa.eu/it/policies/fight-against-terrorism/fight-against-terrorist-financing

https://www.consilium.europa.eu/it/policies/fight-against-terrorismo

https://www.corriere.it/esteri/20_ottobre 29/francia-settantennedecapitato-cattedrale-nizza-14d8028e-19c2-11eb-bdd53 ce 4 cb03ccdf.shtml

https://www.difesa.it/SMD/CASD/IM/CeMiSS/DocumentiVis/Rc erche_da_pubblicare/AI_SA_16_espansionismo_jihadista.pdf

https://www.eda.admin.ch/eda/it/dfae/politica-estera/politica-sicurezza/nuove-sfide/lotta-terrorismo/basi-diritto-internazionalelotta-terrorismo.html

https:/www.ednh.news/it/cronologia-degli-attacchi-terroristici-ineuropa-dal-2004-al-2017/

https://www.giustizia.it/giustizia/it/mg_1_2_1.wp;jsessionid=3595E 0276B2F18BE0E9872FC78BD5935.ajpAL01?facetNode_1 $=0$ _0\&facetNode_4 $=0 \_10 \&$ facetNode_3 $=4$ _59\&facetNode_2 $=1$ $\overline{8}(2015) \&$ previsiousPage $=$ mg_1_2\&contentId $=$ SAN1177293

https://www.gouvernement.fr/radicalisation-les-cinq-grands-axesdu-plan-prevenir-pour-proteger;

https://www.gov.uk/government/publications/counter-terrorismstrategy-contest

https://www.ilpost.it/2020/11/03/attentato-vienna-morti-feriti/

https://www.legislation.gov.uk/ukpga/1998/40/section/1

https:/www.money.it/Isis-non-sconfitto-guerra-terrorismo-nonfinita.

https:/www.notiziegeopolitiche.net/111-settembre-e-le-implicazioni/

https:/www.onuitalia.it/la-strategia-globale-delle-nazioni-unitecontro-il-terrorismo/

https://www.peacelink.it/disarmo/a/335.html

https://www.redattoresociale.it/article/notiziario/bruxelles_i_terroristi_di_seconda_generazione_frutto_delle_politiche_europee

https://www.startinsight.eu/category/europa

https://www.state.gov/foreign-terrorist-organizations/

https://www.un.org/sites/www.un.org.counterterrorism/files/plan action.pdf
Correspondence: Sara Pacileo.

E-mail: sarapacileo2@gmail.com

Key words: Islamic radicalism; criminological profile.

Parole chiave: radicalismo islamico; profilo criminologico.

Palabras clave: radicalismo islámico; perfil criminológico.

Received for publication: 28 September 2020

Accepted for publication: 3 November 2020.

This article is distributed under the terms of the Creative Commons Attribution Noncommercial License (by-nc 4.0) which permits any noncommercial use, distribution, and reproduction in any medium, provided the original author(s) and source are credited.

${ }^{\circ}$ Copyright: the Author(s), 2020

Licensee PAGEPress, Italy

Rivista di Psicopatologia Forense, Medicina Legale, Criminologia 2020: $25: 553$

doi:10.4081/psyco.2020.553 